\title{
Représentations en matière de santé, éducation et soin chez les soignants- éducateurs en éducation thérapeutique du patient : pistes pour la formation
}

\author{
Health, education and care representations among caregivers involved \\ in Therapeutic Patient Education: new avenues for training
}

\author{
Sandrine ROUSSEL, France LIBION et Alain DECCACHE \\ UCL-IRSS-RESO, Éducation Santé Patient, Université catholique de Louvain, Bruxelles, Belgique
}

Manuscrit reçu le 31 août 2010 ; commentaires éditoriaux formulés aux auteurs le 19 février 2011 ; accepté pour publication le 29 mars 2012

\begin{abstract}
Mots-clés
Représentations ; éducation thérapeutique du patient ; soignant ; formation

Keywords Representations; therapeutic patient education; caregiver; training

Résumé - Contexte : La pertinence de l'éducation thérapeutique du patient (ETP) comme complément aux stratégies de soins des problèmes de santé chroniques est avérée. Sa mise en œuvre semble toutefois faire apparaître des incohérences. En psychologie et en pédagogie, l'influence des représentations sur les pratiques professionnelles est globalement connue. But : Cette étude qualitative vise à comprendre les représentations en matière de santé, d'éducation et de soin chez les soignants ainsi que leurs influences sur les pratiques éducatives. Cet article concerne les représentations et leur adéquation avec les modèles biomédical ou biopsychosocial. Méthodes : Des entretiens semi-dirigés ont été réalisés auprès de 30 soignants-éducateurs de Belgique francophone. Une analyse de contenu est réalisée selon un système de catégorisation mixte. Résultats : Certaines représentations non connues jusqu'ici sont mises à jour. Une tendance à un glissement vers des objectifs de santé psychique subjective est observée. Des termes communs apparaissent. Leur acception s'avère toutefois diverse. La concordance (parfaite) des représentations avec un modèle biomédical ou avec un modèle biopsychosocial demeure marginale. Conclusion : Des recherches complémentaires sont nécessaires afin de pouvoir généraliser les résultats. Les formations et un accompagnement adéquat doivent être pensés en vue de favoriser la mise en œuvre de l'ETP.

Abstract - Context: In chronic disease management, the relevance of the Therapeutic Patient Education (TPE) as a complement to health care has been demonstrated. However, implementing it reveals some inconsistencies. In psychology and pedagogy, the weight of representations on professional practices is generally known. Objectives: The qualitative study is designed to understand health, education and care representations among caregivers as well as their impact on educational practices. The paper focuses on representations and their suitability to the (biomedical or biopsychosocial) model. Methods: Semi-structured
\end{abstract}


interviews were conducted with 30 French-speaking caregivers-educators in Belgium. Content analysis was performed and led to a mixed categorization. Results: Several new representations appear and indicate a shift towards subjective mental-health objectives. "Shared" terms emerge but have diverse meanings. A (perfect) match between representations and a single model (biomedical or biopsychosocial) remains marginal. Conclusion: Additional research needs to be carried out to for the results to apply generally. Appropriate coaching and training must be rethought to ensure the implementation of the TPE.

\section{Introduction}

La pertinence de l'éducation thérapeutique du patient (ETP) comme complément aux stratégies de soins dans la prise en charge des problèmes de santé chroniques est avérée. Cette pertinence est accrue du fait du vieillissement de la population et de la hausse potentielle de la prévalence de ce type de pathologie. Le développement de l'ETP passe par la formation qualifiante et le nombre de cursus proposés est en hausse. Malgré ces formations, l'observation de l'exercice de l'ETP fait émerger d'apparentes incohérences. Un diagnostic éducatif peut être non suivi d'une prise en charge formelle des aspects psychosociaux $^{[1]}$. Un hiatus semble apparaître entre les déclarations et les pratiques ; on peut notamment observer: un discours sur l'autonomie du patient coexistant avec une visée d' autonomie thérapeutique, un discours sur l'empowerment cohabitant avec la recherche de l'observance, un discours sur la participation du patient coexistant avec l'exercice de la responsabilité unilatérale du soignant ${ }^{[2]}$. Les représentations sociales pourraient offrir une piste pour comprendre ces divergences. Le lien réciproque entre représentations et pratiques est établi expérimentalement ${ }^{[3,4]}$ et de plus en plus étayé par des recherches sur le terrain ${ }^{[5-8]}$.

L'objectif général de la recherche est de mieux comprendre l'articulation entre représentations et pratiques chez les soignants formés à et pratiquant l'ETP. La définition des représentations sociales adoptée est celle de Jodelet ${ }^{[9]}:$ « une forme de connaissance socialement élaborée et partagée, ayant une visée pratique et concourant à la construction d'une réalité commune à un ensemble social ». L'ETP est définie ici comme un ensemble d'actes éducatifs menés dans le cadre d'une relation soignant-patient, dont la finalité est la santé et/ou la qualité de vie. Son objectif central réside dans l'acquisition de comportements de santé. Les facteurs cognitifs et psychosociaux constituent ses objets initiaux en ce qu'ils influencent directement les comportements de santé ${ }^{[10,11]}$. Ces éléments constituent les objets de recherche en matière de représentations et de pratiques. Cette étude essaye spécifiquement de répondre à la question : Quelle articulation existe-t-il entre les représentations sociales des soignants et leurs pratiques d'ETP ?

Cet article présente un premier volet de résultats : les représentations. Il tente de répondre aux questions suivantes : 1) Quelles sont les représentations actuelles en matière de santé, d'éducation et de soin ?2) Quel est le degré de congruence entre les représentations ? 3) Les éventuelles divergences sont-elles indicatrices de l'évolution vers une acception de l'ETP en adéquation avec le modèle biopsychosocial de santé ?

Les représentations sociales des objectifs de santé, des objectifs de comportements de santé, des objectifs de l'éducation, de la relation de soins et des principes éducatifs sont explorés (Tableau I).

Cette recherche fait partie d'un dispositif de trois recherches menées chacune sur un site distinct. Cet article concerne la recherche menée en Belgique francophone.

\section{Méthodes et population}

Une recherche qualitative et inductive, de type exploratoire est menée. Une enquête transversale par entretiens semi-directifs est conduite auprès d'un échantillon de soignants pratiquant l'ETP.

\section{Population et échantillonnage}

L'échantillonnage vise à approcher un « acteur social compétent ${ }^{[12]}$. Les critères d'inclusion sont : a) être 
Tableau I. Objets de recherche : références opérationnelles.

\begin{tabular}{|c|c|c|c|}
\hline $\begin{array}{l}\text { Catégories } \\
\text { thématiques }\end{array}$ & Définitions & Modalités de catégorisation & $\begin{array}{l}\text { Références } \\
\text { opérationnelles }\end{array}$ \\
\hline $\begin{array}{l}\text { Objectifs de } \\
\text { santé/qualité de } \\
\text { vie }\end{array}$ & $\begin{array}{l}\text { ce que le soignant } \\
\text { cherche à atteindre pour } \\
\text { le patient sur le plan de } \\
\text { la santé }\end{array}$ & $\begin{array}{l}\text { Santé physique/santé globale } \\
\text { (bio-psycho-sociale) }\end{array}$ & Bury ${ }^{[18]}$ \\
\hline $\begin{array}{l}\text { Objectifs de } \\
\text { comportements } \\
\text { de santé }\end{array}$ & $\begin{array}{l}\text { ce que le patient devrait } \\
\text { pouvoir faire en dehors } \\
\text { de la prise en charge par } \\
\text { le soignant }\end{array}$ & Auto-soins/adaptation & $\operatorname{HAS}^{[19]}$ \\
\hline $\begin{array}{l}\text { Objectifs de } \\
\text { l'éducation }\end{array}$ & $\begin{array}{l}\text { ce que le patient devrait } \\
\text { acquérir lors de } \\
\text { l'éducation. } \\
\text { Comprend les types de } \\
\text { savoirs visés et les } \\
\text { objets d'éducation }\end{array}$ & $\begin{array}{l}\text { (types de savoirs visés) savoir et savoir- } \\
\text { faire/objectifs multiples (compétences, } \\
\text { motivation, facteurs psychosociaux et } \\
\text { psychologiques) } \\
\text { (objets) maladie et traitement/santé maladie } \\
\text { et connaissance de soi }\end{array}$ & $\begin{array}{l}\text { Deccache }^{[20]} \\
\text { Taylor }^{[21]}\end{array}$ \\
\hline $\begin{array}{l}\text { Relation } \\
\text { soignant- } \\
\text { soigné }\end{array}$ & $\begin{array}{l}\text { comprend le mode de } \\
\text { relation, le rôle du } \\
\text { soignant et le rôle du } \\
\text { patient }\end{array}$ & $\begin{array}{l}\text { (décision) observance/alliance } \\
\text { thérapeutique } \\
\text { (activités) patient passif et soignant actif/ } \\
\text { participation mutuelle } \\
\text { (échange d'info) information/ } \\
\text { communication } \\
\text { (attitude en situation extrême de refus de } \\
\text { suivi de traitement) ignore/entend/ } \\
\text { comprend/accepte/ } \\
\text { soutient }\end{array}$ & $\begin{array}{l}\text { Deccache }^{[10]} \\
\text { Szasz \& Hollender }{ }^{[22]} \\
\text { Deccache \& } \\
\text { Lavenhomme }^{[23]}\end{array}$ \\
\hline $\begin{array}{l}\text { Principes } \\
\text { éducatifs }\end{array}$ & $\begin{array}{l}\text { la manière dont le } \\
\text { soignant s'y prend pour } \\
\text { éduquer }\end{array}$ & $\begin{array}{l}\text { Enseignement/apprentissage } \\
\text { Accompagnement éducatif simple/ } \\
\text { accompagnement psychosocial } \\
\text { Démarche non personnalisée, commune à } \\
\text { tous les patients/démarche personnalisée }\end{array}$ & $\begin{array}{l}\text { Bélair }^{[24]}, \text { Jouquan \& } \\
\text { Bail }^{[25]} \\
\text { Deccache \& } \\
\text { Lavenhomme }^{[23]} \\
\text { De Ketele \& } \\
\text { Roegiers } \\
{[26], \mathrm{WHO}^{[11]}}\end{array}$ \\
\hline
\end{tabular}

HAS : Haute autorité de santé. WHO : World Health Organization.

soignant ; b) pratiquer l'ETP ; c) être formé à l'ETP ; d) travailler en équipe et e) accepter de participer à la recherche. L'acception de l'ETP est étendue à un champ plus vaste que la prise en charge de la maladie chronique. Dans tous les cas, une « chronicité » dans la relation soignant-patient est présente. La procédure d'échantillonnage prévoit la constitution d'une liste de répondants potentiels sur base d'avis d'experts, provenant d'organisations professionnelles actives en ETP. L'échantillon est constitué des premiers soignants contactés, en mesure de participer. La saturation des données a été atteinte au $30^{\mathrm{e}}$ entretien. Trente-huit soignants ont été contactés ; huit n'ont pu être retenus car injoignables $(n=3)$, ne souhaitant pas participer à la recherche $(n=2)$ ou n'exerçant plus l'ETP $(n=3)$. 


\section{Outils}

Un guide d'entretien et un questionnaire d'identification sont conçus pour les besoins de la recherche. Le guide d'entretien élaboré à partir du cadre théorique est affiné après la pré-enquête. Le guide comprend une consigne de départ (exemple : Parlez-moi de ce que vous faites actuellement au niveau de l'éducation avec le patient ?), les thèmes à évoquer et des suggestions de questions de relance :

- objectifs de santé (ou de qualité de vie) : Que visez-vous pour le patient sur le plan de la santé?

- Comportements de santé : Qu'est-ce que le patient devrait faire? Qu'attendez-vous que le patient fasse en dehors de la prise en charge?

- Objectifs de l'éducation : Qu'est-ce qu'il devrait savoir/devrait pouvoir faire?

- Relation soignant-soigné : Pour vous, c'est quoi un patient «difficile»? Des patients disent « je ne veux plus prendre mon traitement, je m'en fous de vivre si c'est pour vivre comme cela ». Que faitesvous?

- Principes éducatifs : Pour vous, éduquer c'est ...

Le questionnaire d'identification permet de recueillir des données relatives au parcours du répondant en ETP (c.-à-d. nombre et type de service(s), nombre de projets, type d'organisation, implication dans des réseaux, profession, formations complémentaires, ancienneté en ETP, organisation des soignants, approche - individualisée et/ou collective - des patients, proportion des activités dédiées à l'ETP, existence d'un soutien méthodologique et âge). Il comprend 15 questions.

\section{Dispositif de recueil}

Les données sont recueillies par questionnaire d'identification auto administré et entretien semi-directif. Ces deux outils sont testés lors d'une pré-enquête. L'entretien se clôture lorsque toutes les catégories du guide sont abordées. La durée moyenne d'un entretien est de 68 minutes. Tous les entretiens sont enregistrés et retranscrits intégralement.
Traitement et analyse des données

Les « unités de sens » sont extraites des retranscriptions et encodées dans une grille Excel sur logiciel Microsoft. Une unité de sens est tout extrait du discours (phrase ou partie de phrase) permettant de catégoriser le positionnement du répondant par rapport aux objets de recherche. Une analyse de contenu est réalisée selon un système de catégorisation mixte, associant catégories prédéfinies (objets de recherche) et catégories émergentes.

\section{Validité}

Un dispositif vise à assurer la validité tout au long de la recherche. Les entretiens de la pré-enquête sont menés simultanément par deux chercheurs. La validité de l'analyse et de la signification attribuée aux verbatims est assurée par la triangulation des chercheurs. Des réunions d'intervision sont organisées régulièrement par site et en commun avec les autres équipes. Un retour aux acteurs est également prévu. Les participants sont sollicités pour une séance de feed-back collective afin de recueillir leur analyse et leur interprétation.

\section{Résultats}

L'échantillon comprend 19 femmes et 11 hommes. Treize répondants sont âgés de 40 à 49 ans, sept de 50 à 59 ans, deux de plus de 60 ans et cinq de moins de 40 ans. Ces soignants sont infirmiers $(n=16)$, médecins $(n=7)$, ergothérapeutes $(n=2)$, kinésithérapeutes $(n=2)$, psychologues $(n=1)$, assistants sociaux $(n=1)$ ou éducateurs $(n=1)$. Ils exercent dans des hôpitaux ( $n=19)$, à domicile ou en cabinet privé $(n=9)$, dans des maisons médicales $(n=2)$ ou de la naissance $(n=1)$. Dix-huit soignants sont spécialisés dans un ou plusieurs domaine(s) (assuétudes, diabète, dialyse, oncologie, obstétrique, gériatrie, soins palliatifs). Douze répondants exercent comme médecin généraliste ou coordinateur. Les formations en ETP 
sont des masters $(n=6)$, des formations de l'enseignement supérieur non universitaire $(n=4)$ et des formations courtes $(n=14)$. Onze formations sont spécifiquement dédiées à l'ETP.

Quatorze répondants estiment consacrer au minimum $50 \%$ de leur temps de travail à l'ETP. L'ETP est pratiquée par 12 soignants depuis 20 ans et plus, par sept soignants depuis moins de 10 ans. L'ETP se réalise essentiellement en entretien en face-à-face avec un patient $(n=22)$. Plus rarement $(n=6)$, cette approche individuelle est combinée à une approche groupale. Les soignants opèrent seuls $(n=12)$, en équipe $(n=4)$ ou seuls et en équipe $(n=13)$. Tous pratiquent l'intervision, soit un échange entre professionnels relatif aux pratiques, en vue de leur amélioration.

Représentations des objectifs de santé et de qualité de vie

L'ETP vise le maintien ou l'amélioration de la santé des patients. Il s'agit de déterminer ce que le soignant déclare viser pour ses patients. Les catégories prédéfinies prévoient une vision en termes de santé globale (modèle biopsychosocial) ou santé physique (modèle biomédical). Vingt-six répondants rapportent viser la santé globale, trois la santé physique uniquement. La «santé globale» recouvre toutefois des acceptions variées. Les types de santé englobés (physique, psychique et/ou sociale) diffèrent parmi les répondants. L'acception la plus générique est la qualité de vie, voire un projet de société.

Une catégorie émerge : le bien-être ou la santé psychique subjective. Il ne s'agit pas de la santé biopsychosociale (soit la santé physique, psychologique et sociale, pouvant toutes être déclinées selon une dimension objective et subjective) mais une partie de celle-ci : la santé psychologique dans sa dimension subjective. Dans ces cas, la santé physique n'est pas visée: «Le bonheur, pas la santé physique, parce que le bonheur n'est pas dans la santé ». La santé sociale est parfois présentée comme relevant de la compétence d'autres soignants. En considérant la catégorie « santé psychique subjective », trois soignants visent la santé physique, 15 la santé psychique subjective, 11 la santé globale.
Représentations des objectifs de comportements de santé

L'ETP vise l'adoption de comportements (de santé) adéquats à l'état de santé du patient. Il s'agit de déterminer ce que le soignant pense que le patient devrait faire pour sa santé ou son traitement. Les catégories prévoient les auto-soins (c.-à-d. compétences de sécurité et de prise en charge autonome de la maladie ou du traitement) et l'adaptation (c.-à-d. compétences d'autosoins et d'action sur les facteurs psychosociaux d'ajustement à la maladie). Vingt et un soignants disent viser l'adaptation ; six visent les auto-soins.

Les objectifs d'auto-soins cités sont : " se conformer à la prescription », « réaliser son auto-dialyse », ou « se protéger contre les risques de rechute». Pour certains soignants, les objectifs évoluent en fonction du temps restant de prise en charge. L'adaptation à la maladie fait place à des objectifs d'auto-soins lorsque la fin de la prise en charge approche. "Digérer sa stomie » puis « Changer sa poche ». Les objectifs d'adaptation sont exprimés selon deux optiques distinctes : a) être acteur : « Comprendre pour être responsable »; b) accepter sa maladie : "Accepter d'avancer, sans renoncer à vivre ». L'adaptation peut être visée, sans que les auto-soins ne soient évoqués. Le comportement de santé est alors «poser des choix justes pour soi ».

L'autonomie est évoquée spontanément par 26 soignants à la question de ce qui est visé pour le patient. Une distinction est opérée entre autonomie fonctionnelle (c.-à-d. le savoir-faire que le patient déploie pour gérer sa maladie ou sa santé) et autonomie décisionnelle (c.-à-d. la capacité du patient de poser des choix en matière de santé). L'autonomie fonctionnelle seule n'est pas la pleine autonomie si elle s'inscrit dans un programme défini par le soignant $^{[13]}$. Seule l'autonomie décisionnelle correspond à un comportement d'adaptation psychosociale. Douze répondants visent l'autonomie fonctionnelle, cinq l'autonomie décisionnelle et huit les deux types d'autonomie (Tableau II).

\section{Représentations des objectifs de l'éducation}

Les objectifs de l'éducation consistent en ce que le patient devrait acquérir par l'éducation. Ils comprennent 
Tableau II. Représentations des objectifs de comportements de santé (autonomie) (numéro d'identification des soignants).

\begin{tabular}{|l|l|l|}
\hline Autonomie fonctionnelle uniquement & $\begin{array}{l}\text { Autonomie décisionnelle } \\
\text { uniquement }\end{array}$ & $\begin{array}{l}\text { Autonomie fonctionnelle et } \\
\text { autonomie décisionnelle }\end{array}$ \\
\hline $\begin{array}{l}1,8,9,13,15,16,19,20,23,25,26,27 \\
(n=12)\end{array}$ & $\begin{array}{l}2,5,6,7,10 \\
(n=5)\end{array}$ & $\begin{array}{l}3,11,14,18,21,22,24,30 \\
(n=8)\end{array}$ \\
\hline en fonction du service $: 17(n=1)$, non pertinent $: 4,12,28,29(n=4)$ & \\
\hline
\end{tabular}

Tableau III. Représentations des types de savoir visés.

\begin{tabular}{|l|l|l|}
\hline $\begin{array}{l}\text { Savoirs et savoir-faire } \\
\text { uniquement }\end{array}$ & $\begin{array}{l}\text { Objectifs multiples sans les } \\
\text { savoirs et savoir-faire }\end{array}$ & Objectifs multiples \\
\hline $14,19,20,23,25$ & 9,10 & $\begin{array}{l}1,2,3,4,5,6,7,11,12,13,15,16,18,21, \\
22,24,27,30 \\
(n=18)\end{array}$ \\
$(n=5)$ & $(n=2)$ & \\
\hline En fonction $: 8,17,26(n=3)$, absence de réponses $: 28,29(n=2)$
\end{tabular}

Tableau IV. Représentations des objets d'éducation.

\begin{tabular}{|l|l|l|}
\hline Maladie et traitement uniquement & $\begin{array}{l}\text { Connaissance de soi } \\
\text { uniquement }\end{array}$ & Tous \\
\hline $\begin{array}{l}1,4,7,8,12,13,14,19,20,23,24,25,26 \\
(n=13)\end{array}$ & $\begin{array}{l}3,6,10 \\
(n=3)\end{array}$ & $\begin{array}{l}2,5,9,11,15,16,18,21,22,27,30 \\
(n=11)\end{array}$ \\
\hline En fonction : $17(n=1)$, absence de réponse $: 28,29(n=2)$ & \\
\hline
\end{tabular}

deux dimensions : le(s) type(s) de savoir(s) visé(s) (c.à-d. les modalités de savoirs, la motivation, les facteurs psychosociaux) et les objets de l'éducation (c.-à-d. les objets sur lesquels s'exercent l'acquisition de savoirs : le traitement, la maladie, la connaissance de soi).

Concernant le type de savoirs visés, les catégories thématiques prévoient les savoirs (S) et savoir-faire (SF) et les savoirs multiples (c.-à-d. les S, SF, savoirêtre ainsi que les méta-savoirs : la motivation, les facteurs psychosociaux). Vingt soignants annoncent viser des savoirs multiples, cinq des $\mathrm{S}$ et $\mathrm{SF}$ uniquement. Le positionnement de trois soignants fluctue entre ces deux modalités en fonction du type de service ou du temps restant de prise en charge. Une catégorie émerge : les savoirs multiples sans les $\mathrm{S}$ et $\mathrm{SF}$ : des objectifs de savoir-être (SE) sont évoqués (Tableau III). Le SE est annoncé comme essentiel par 17 soignants.
Concernant les objets d'éducation, les catégories thématiques prévoient la maladie et le traitement ainsi que tous les objets d'éducation (c.-à-d. la santé, la maladie, la connaissance de soi). Treize répondants annoncent la maladie et le traitement, 11 tous les objets, trois la connaissance de soi uniquement. «Leur redonner confiance en elles, en leurs corps... Ça fait partie d'un retour de leur confiance en elles » (Tableau IV).

\section{Représentations de la relation soignant-patient}

Les représentations de la relation soignant-patient comprennent la manière d'envisager cette relation, les rôles des deux protagonistes : le soignant et le patient ainsi que l'attitude en situation extrême. 
Tableau V. Représentations de la relation de soignant-patient (« décision » et activités).

\begin{tabular}{|l|l|l|}
\hline Activités & Décision & Observance \\
\hline Activité/passivité & $\begin{array}{l}1,4,8,19,20,26 \\
(n=6)\end{array}$ & $(n=0)$ \\
\hline Participation mutuelle & $\begin{array}{l}7,16,23,25,27 \\
(n=5)\end{array}$ & $\begin{array}{l}2,3,5,6,9,10,11,12,14,15,18,21,22,24,28,29,30 \\
(n=17)\end{array}$ \\
\hline
\end{tabular}

\section{Relation soignant-patient}

Celle-ci est analysée selon trois dimensions : le mode de décision, la participation aux activités et l'échange d'information.

Le mode de décision concerne la place que les soignants déclarent laisser au patient dans la prise de décision. Les catégories thématiques prévoient l'alliance thérapeutique (c.-à-d. le patient participe à la décision, négocie) et l'observance (c.-à-d. le patient est passif). Dix-sept soignants se positionnent dans un modèle d'alliance thérapeutique, 11 dans un modèle d'observance. Deux soignants deviennent moins coercitifs lorsque le patient ne peut suivre le traitement standard : «S'ils ne peuvent plus suivre, on leur permet de lâcher un peu de lest ».

La participation aux activités s'intéresse à la question de savoir comment le soignant conçoit l'implication du patient dans les activités. Les catégories thématiques prévoient la participation mutuelle (c.-à-d. le patient et le soignant sont acteurs) et la passivité/activité (c.-à-d. le patient est passif-le soignant est actif). Vingt-deux répondants se placent dans un modèle de participation mutuelle, six dans un modèle de passivité/activité (Tableau V).

L'échange d'information se décline en un modèle de communication (privilégiant les échanges, la communication empathique et l'écoute du patient) et un modèle d'information (favorisant peu l'expression du patient). Vingt-six soignants se positionnent dans un modèle de communication, deux dans un modèle d'information.

\section{Rôle du soignant}

Dix répondants citent «Accompagner » pour qualifier leur rôle. Des expressions voisines sont utilisées $(n=3)$ : «faciliter, soutenir, être la main qui aide». Ces termes sont posés comme alternatives à «éduquer », qui n'a pas toujours un écho favorable: «Je pense qu'on a un rôle éducatif, mais je ne me sens pas éducateur».

Le degré idéal d'implication émotionnelle du soignant varie d'important $(n=6)$, à nul $(n=1)$ en passant par certaines limites $(n=5)$. «Faire son travail avec amour et passion » (implication importante), «Je ne me sens pas responsable de leur santé sinon je deviens fou » (implication nulle).

\section{Rôle du patient}

L'exploration du rôle du patient est menée en sondant les cas extrêmes : le « patient modèle » et le «patient difficile ». Ce dispositif vise à amener le répondant à se positionner.

Le «patient modèle » fait apparaitre deux catégorisations : le degré d'implication attendu du patient et ses qualités.

Eu égard au degré d'implication, le patient modèle est actif ( $n=18)$ ou à l'inverse obéissant et docile $(n=5)$. Les qualités du patient sont analysées selon ce qu'il est (c.-à-d. les caractéristiques de personnalité qui font qu'il puisse mener à bien son traitement ou la relation de soins) et ce qu'il fait (c.-à-d. tout ce qu'il met en place sur le plan du comportement ou des attitudes dans cet objectif). Six soignants se prononcent selon la modalité « fait», sept selon la modalité « est », 10 selon les deux modalités. La modalité « fait » se décline de compliant à réellement participatif : "Prend en compte ce qu'il a appris, les conseils » (compliant), "Interpelle le soignant avec des questions pertinentes, en lui demandant de l'aide » (participatif). La modalité « est » comprend des traits de 
Tableau VI. Typologie de l'attitude du soignant en situation extrême de refus de soins.

\begin{tabular}{|c|c|c|c|c|c|c|}
\hline 0 & \multicolumn{2}{|l|}{ Ignore } & \multicolumn{3}{|c|}{ «je n’entends pas » } & \\
\hline 1 & \multicolumn{2}{|c|}{ Entend } & \multicolumn{3}{|c|}{ «j'entends mais je ne comprends pas » } & \\
\hline 2 & \multicolumn{2}{|c|}{ Comprend } & \multicolumn{3}{|c|}{ «j'entends, je comprends, (je respecte) mais je n'approuve pas » } & \\
\hline 3 & \multicolumn{2}{|c|}{ Accepte } & \multicolumn{4}{|c|}{$\begin{array}{l}\text { «j'entends, je comprends, j'approuve mais n'aide pas » } \\
\text { humanisme fataliste : «j'entends et approuve sans qu'il ne me soit nécessaire de } \\
\text { comprendre ». }\end{array}$} \\
\hline 4 & \multicolumn{2}{|c|}{ Soutient } & \multicolumn{4}{|c|}{ «j'entends, je comprends, j’approuve, j'aide activement » } \\
\hline \multirow{2}{*}{\multicolumn{2}{|c|}{$\begin{array}{c}\text { Attitude du } \\
\text { soignant }\end{array}$}} & ignore & entend & comprend & accepte & soutient \\
\hline & & $\begin{array}{l}20,23 \\
(n=2)\end{array}$ & $\begin{array}{c}25 \\
(n=1)\end{array}$ & $\begin{array}{l}1,7,8,11,21 \\
27 \\
(n=6)\end{array}$ & $\begin{array}{c}3,4,6,13,18,19,24,29 \\
(n=8) \text { dont humanisme fatalism } \\
4,6,13,19,24(n=5)\end{array}$ & $\begin{array}{l}2,5,9,10,12,14 \\
22,28,30 \\
(n=9)\end{array}$ \\
\hline
\end{tabular}

personnalité et l'attitude vis-à-vis du traitement: «Non agressif et non revendicateur, ouvert et de bonne composition » (traits de personnalité), « Satisfait de la relation, de lui-même et du bénéfice apporté » (attitude).

Le « patient difficile » a les qualités inverses du « patient modèle » ou ne respecte pas le soignant dans son rôle. Parmi les qualités « inverses », la modalité du patient passif est très présente. Elle confronte le soignant à l'impuissance. «Celui qui n'a pas compris qu'il est le maître de sa santé », " celui qui met tout et tous en échec ». À l'instar du " patient modèle», une catégorisation peut être établie en fonction de ce qu'il est ou fait. Treize soignants se prononcent selon la modalité «fait», deux selon la modalité «est», trois selon les deux modalités.

Concernant l'objet sur lequel se focalise la représentation, celle du «patient modèle » se focalise davantage sur le patient (12 sont centrées vers le patient, cinq sur le soignant). Celle du «patient difficile » se focalise davantage sur le soignant (12 sont centrées sur le patient, neuf sur le soignant).

Certains soignants identifient des patients plus ou moins « éducables » en fonction de leur formation ou leur intelligence et le type de pathologie. Un haut niveau de formation peut être perçu comme favorisant $(n=1)$ ou comme entravant $(n=3)$ l'ETP. «Tout ce quifavorise l'éducation, favorise aussil'éducation du patient»(favorisant). "Des gens au-dessus de la moyenne qui ne veulent pas entendre parler de tout cela et qui s'enfoncent $\gg$ (entravant). Deux catégories de patients sont identifiées comme difficilement éducables : les diabétiques et les toxicomanes.

\section{Attitude en situation extrême}

Cette représentation (c.-à-d. le refus par le patient de suivre le traitement, au péril de sa vie) est explorée afin de sonder les intentions du soignant au-delà du discours de respect du patient. Cette typologie (Tableau VI) est cumulative. Les niveaux successifs «ignore, entend, comprend, accepte, soutient» englobent les précédents. Deux soignants ignorent ce que le patient dit : "L'équipe lâche rarement. On sait $q u$ 'on peut soigner, on veut soigner ». Un entend : «On n'écoute pas le patient qui dit j'ai pas envie». Six comprennent: "le choix du patient, c'est la théorie, parce que je me sentirais mal si le patient revient en acidocétose ». Huit acceptent : « Si la personne est capable de décider ce qu'elle veut ou pas de sa vie, on respecte cela ». Neuf soutiennent : "Je suis très bien avec un patient qui ne fait pas ce qu'il faut pour aller mieux. J'y reviendrai (à la cessation tabagique) puisque je prescrirai des médicaments pour qu'elle 
Tableau VII. Représentations des principes éducatifs.

\begin{tabular}{|l|l|}
\hline Enseignement & Apprentissage \\
\hline $\begin{array}{l}4,6,11,13,20,27 \\
(n=6)\end{array}$ & $\begin{array}{l}1,2,3,5,7,8,9,10,12,14,15,18,19,22,23,24,25,26 \\
(n=18)\end{array}$ \\
\hline \multicolumn{2}{|l|}{ En fonction (de la pathologie) $16,17,21(n=3)$. Non-pertinent $: 28,29,30(n=3)$} \\
\hline
\end{tabular}

ait moins d'inconfort du fait qu'elle continue à fumer ». La représentation de l'attitude de trois soignants fluctue en fonction du type du service ou du type de demande du patient : un soignant comprend en orthopédie mais soutient en soins palliatifs. Dans la relation de soins, un autre entend la demande mais ne l'écoute pas et soutient la décision d'en finir.

Une catégorie apparaît : à côté de l'acceptation, apparait l' « humanisme fataliste », expression empruntée à Garcia Marquez ${ }^{[14]}$. L'humanisme fataliste est la conception selon laquelle " chaque homme est maître de sa propre mort. La seule chose que nous (médecins) pouvons faire est l'aider à mourir sans peur, ni douleur ». L'acceptation concerne trois soignants. L' « humanisme fataliste » en concerne cinq. Dans ce cas, le soignant accepte parce qu'il ne peut rien y faire, que le patient est en droit d'exercer sa liberté de choix, en maître de sa destinée. Ce type d'acceptation n'implique pas le niveau qui précède : la compréhension. Elle est plutôt abdication : « Ce n'est pas grave si la personne ne le fait pas, c'est sa vie».

\section{Représentations des principes éducatifs}

Les principes éducatifs concernent la manière dont le soignant s'y prend pour éduquer. Les catégories thématiques prévoient deux dimensions : un modèle d'enseignement $v s$. d'apprentissage et un accompagnement éducatif « simple»vs. «psychosocial».

Le modèle d'apprentissage est centré sur l'apprenant et les acquis. Le modèle d'enseignement est centré sur l'enseignant et les contenus à transmettre. Dixhuit répondants se placent dans un modèle d'apprentissage, six dans un modèle d'enseignement (Tableau VII).

L'accompagnement éducatif «simple» fait référence à des modèles de pratiques fondés sur la motivation, la facilitation des apprentissages et la réappropriation des SF. L'accompagnement éducatif « psychosocial » fait référence à des modèles de pratiques fondés sur la relation de confiance, la gestion des émotions, la démarche de projet et la résolution de problème. Vingt soignants se placent dans une perspective « psychosociale », cinq dans une perspective « simple ».

Peu de répondants $(n=4)$ présentent des représentations en cohérence parfaite avec un modèle de santé unique (c.-à-d. cohérent avec le modèle biomédical ou biopsychosocial sur les représentations relatives aux objectifs de santé, aux objectifs de comportement de santé, aux types de savoir et aux objets de savoir). Un répondant présente des représentations cohérentes avec le modèle biomédical. Trois présentent des représentations cohérentes avec un modèle biopsychosocial. Vingt et un présentent des représentations hétérogènes en regard de ces deux modèles.

\section{Discussion}

La majorité des soignants formule des objectifs de santé en accord avec la santé globale. Une seconde analyse fait toutefois apparaître une forme de santé en opposition à la santé physique objective : la santé psychique subjective. Cela soulève plusieurs questions : S'agit-il d' «oubli » de la santé physique parce que «évidente» pour le soignant ? S'agit-il d'une progression vers le modèle biopsychosocial ou un renoncement à celui-ci ? S' agit-il d'un abandon complet du modèle biomédical ? Quels en sont les risques ? Y-a-t-il risque d' « abdication » du rôle professionnel (et des responsabilités) du soignant au profit d'une liberté maximale du patient ? Comment la participation reste-t-elle possible s'il y a suppression d'un des acteurs de la négociation : le soignant? 
L'implication du patient dans la relation soignant-patient est plus fréquente dans la participation aux activités que dans les décisions qui fixent le cadre de ces activités. Cinq soignants ne donnent pas de marge dans la décision mais bien dans l'implication dans les activités. La participation est-elle possible sans codécision ? Ce positionnement ne s'inscrit pas dans le modèle biopsychosocial. La codécision fait partie intégrante des principes d'apprentissage, du développement de capacités psychosociales.

Concernant l'attitude en situation extrême, on touche à l'empathie vis-à-vis du patient. L'humanisme fataliste traduit-il un déficit d'empathie ?Comment le soignant peut-il approuver sans comprendre ce que l'autre communique et ressentir ce que ce refus suscite en lui ? Lorsque le soignant accepte le choix du patient en comprenant, il va plus souvent le soutenir. Avec l'humanisme fataliste, l'acceptation est intellectuelle et non suivie d'acte.

Le degré idéal d'implication émotionnelle du soignant est très variable, allant d'une implication importante à la nécessité de mettre des limites. Ces deux tendances se retrouvent dans la littérature. Parsons ${ }^{[15]}$ prône, dans son modèle fonctionnel, la neutralité affective du soignant (caractérisée par un rapport objectif et non-émotionnel) alors que Menninger ${ }^{[16]}$ défend l'idée que le caring fait partie de la qualité d'un système de soins. Observe-t-on une évolution vers plus d'investissement émotionnel? Les soignants investis émotionnellement présentent un tableau plus concordant sur le plan de la participation. À l'exception d'un, tous présentent un modèle de participation mutuelle, soit un positionnement inverse de celui du patient passif attendu par Parsons. La nécessité soulignée de limites dans l'implication serait-elle une difficulté de gérer l'émotionnel ou simplement une position cohérente avec le principe de neutralité affective ? S' agit-il d'une difficulté de percevoir les limites du rôle attendu d'un soignant-éducateur ?

La représentation de patients plus ou moins « éducables » témoigne de la persistance d'une vision de l'ETP nécessitant la motivation préalable du patient et n'incluant pas le travail sur la motivation comme un objectif pédagogique.

Les représentations du « patient modèle » concernent de façon égale « ce qu'il fait » et « ce qu'il est ».
Les représentations $\mathrm{du}$ « patient difficile » s'attachent essentiellement à « ce qu'il fait ». Ces dernières renvoient davantage à des causes situationnelles (c.-à-d. une lecture en termes de rôles sociaux) qu'à des causes dispositionnelles $^{[17]}$ (c.-à-d. une lecture en termes d'humeur, personnalité, valeurs et intentions). Cette lecture en termes situationnels témoigne-t-elle du fait que le soignant se sente investi du rôle de modifier les comportements? Une lecture du «patient difficile» en termes dispositionnels peut réduire la marge d'action du soignant, tandis qu' une lecture en termes de rôle le place en position d'infléchir la situation. L'observation de l'objet sur lequel se centre la représentation va dans le sens de la responsabilité du soignant. Les représentations du «patient modèle» s'axent essentiellement sur le patient, celles du « patient difficile » s'axent sur le soignant. Les déclarations témoignent d'une gratification du soignant lorsqu'il parvient à mener à bien sa mission éducative. "J'ai à la limite plus de plaisir quand j'ai un patient que j'arrive à faire bouger un peu ». Les verbatims traduisent aussi un sentiment de « souffrance » chez les soignants $(n=6)$ qui ne parviennent pas à mener leur mission éducative. Ces verbatims font état de problèmes d'incompréhension, d'impuissance et de non respect en tant que personne.

Peu de répondants présentent des représentations en cohérence avec un modèle de santé unique (biomédical ou biopsychosocial). La majorité des répondants présentent des représentations hétérogènes en regard des modèles. Cette hétérogénéité des représentations est-elle indicative d'une évolution vers le modèle biopsychosocial ? Une tendance semble corroborer cette hypothèse. Lorsque l'hétérogénéité des représentations est observée, les combinaisons les plus courantes comprennent systématiquement des représentations en cohérence avec le modèle biopsychosocial. Lorsque le niveau de représentations devient plus opérationnel, les représentations relatives à l'ETP s'éloignent du modèle biopsychosocial chez la plupart des soignants qui rapportent des représentations hétérogènes. Cette tendance est-elle un indice d'un hiatus entre souhaité et vécu ? Quels sont les éléments (absence d'outils opérationnels, obstacles organisationnels...) qui entravent la mise en œuvre de l'ETP? 
La recherche présente différentes limites : 1) les représentations sont explorées au moyen du discours. Or, le langage ne permet l'expression que d' une partie seulement des représentations; 2) les catégories émergentes n'ont pu être explorées auprès de l'ensemble des répondants ; 3 ) parmi les 30 soignants interviewés, 22 soignants pratiquent l'éducation du patient en face-à-face uniquement. Aucun soignant ne pratique cette éducation auprès de groupes uniquement ; 4) les chercheurs sont issus d'une équipe de recherche en éducation du patient ce qui peut avoir induit des réponses favorables à cette approche en raison de la désirabilité sociale.

\section{Conclusion}

Cette recherche montre l'existence de représentations non connues jusqu'ici : la santé psychique subjective, l'adaptation psychologique sans auto-soins, l'autonomie décisionnelle sans autonomie fonctionnelle, l'importance du SE, la connaissance de soi sans celle de la maladie, l'humanisme fataliste, .... Elle souligne que l'emploi de termes communs tels que autonomie, accompagnement, santé globale, participation, éducation, ... peut cacher des acceptions très diverses. La concordance parfaite des représentations avec un modèle de santé unique (biomédical ou biopsychosocial) demeure marginale.

Il y a matière à poursuivre les recherches afin de déterminer l'incidence des représentations sur les pratiques ainsi que la mesure dans laquelle la tendance émergente, la santé psychique subjective, constitue un nouveau modèle. Ces incidences sont particulièrement intéressantes dans le cadre des pratiques d'équipe et des messages, éventuellement contradictoires, qui parviennent au patient.

Sur le plan des activités de formation et d'aide à l'action, l'hétérogénéité des représentations en matière d'ETP invite à : 1) développer des cursus en s'inspirant d'une forme d'ETP construite sur les représentations des soignants et à inférer. Cette méthode constituerait l'objet de formation et permettrait d'assurer la cohérence des méthodes de formation avec celles de l'ETP ; 2) envisager la cohérence entre l'approche recommandée en formation et le contexte qui cadre la pratique ; 3 ) veiller à l'intégration des identités de soignant et d'éducateur afin qu'une identité ne se construise pas au détriment de l'autre ; 4) encourager l'intervision afin de construire l'ETP en actant la diversité de ses modalités d'application. L'intervision encouragerait le partage des représentations et favorisera l'échange sur le sens donné aux vocables propres à la discipline.

\section{Contributions}

Tous les auteurs ont participé à la conception du protocole de recherche. France Libion et Sandrine Roussel ont mené le recueil et le traitement de données. Sandrine Roussel a procédé à l'analyse qualitative et à la rédaction de l'article. Alain Deccache a dirigé la recherche et apporté une aide à la rédaction.

\section{Remerciements}

Nous remercions Cyril Crozet*, Rémi Gagnayre*, Anne Le Rhun** et Pierre Lombrail** pour leur collaboration (*Université Paris 13. ** CHU de Nantes).

\section{Financement}

Ce travail de recherche a été réalisé sur fonds propres de l'Université catholique de Louvain et avec le soutien de l'Institut national de prévention et d'éducation pour la santé (INPES), Paris.

\section{Références}

1. Le Rhun A, Greffier C, Lamour P, Gagnayre R, Lombrail P. État des lieux en 2006 de 16 programmes d'éducation thérapeutique au CHU de Nantes (et réseaux rattachés) : une démarche éducative centrée sur le patient? Éducation du patient et Enjeux de santé. 2007;25:17-21. 
2. Deccache A, van Ballekom K. From patient compliance to empowerment and consumer's choice: Evolution or regression? An overview of patient education in French speaking European countries. Patient Educ Couns 2010;78:282-7.

3. Abric J-C, editor. Pratiques et représentations sociales. $4^{\mathrm{e}}$ ed. Paris : PUF-Psychologie sociale ; 2003.

4. Seca J-M. Les représentations sociales. Paris : Armand Colin ; 2005.

5. Bishop G. Les prototypes de la maladie : nature et fonctions. In : Petrillo G, editor. Santé et société. La santé et la maladie comme phénomènes sociaux. Lausanne : Delachaux et Niestlé 2000:45-66.

6. Jodelet D, Ohana J. Représentations sociales de l'allaitement maternel : une pratique de santé entre nature et culture. In : Petrillo G, editor. Santé et société. La santé et la maladie comme phénomènes sociaux. Lausanne : Delachaux et Niestlé 2000:3766.

7. Jourdan D, Piec I, Aublet-Cuvelier B, Berger D, Lejeune M, Laquet-Riffaud A, et al. Education à la santé à l'école : pratiques et représentations des enseignants du primaire. Santé publique 2002;4:40323.

8. Vega A. Les infirmières hospitalières françaises : l'ambiguiité et la prégnance des représentations professionnelles. Sci Soc Santé 1997;15:103-41.

9. Jodelet D, editor. les représentations sociales. Paris : PUF ; 1989.

10. Deccache A. Un cadre théorique pour comprendre et agir sur le problème de la non-compliance aux traitements. Bulletin d'éducation du patient 1995;14:612.

11. WHO Europe. Therapeutic Patient Education. Continuing education programmes for healthcare providers in the field of prevention of chronic diseases. Report of a WHO working group. 11-14 June 1997. Copenhagen: WHO Regional Office for Europe ; 1998.

12. Savoie-Zajc L. Comment peut-on construire un échantillonnage scientifiquement valide? Recherches qualitatives, Hors série, 2007;5:99-111.

13. Malherbe J. Sujet de vie, objet de soins ? Montréal : FIDES ; 2007.
14. Garcia Marquez G. L'amour aux temps du choléra. Paris : Grasset ; 1985.

15. Parsons T. The social system. Glencoe : The free press ; 1951.

16. Menninger W. "Caring" as Part of Health Care Quality. JAMA 1975;234:836-7.

17. Fiske ST. Psychologie sociale. Bruxelles : De Boeck; 2008.

18. Bury J. Education pour la santé. Concept, enjeux, planification. Bruxelles : De Boeck Université ; 1988.

19. Haute Autorité de Santé. Éducation thérapeutique du patient. Définitions, finalités et organisation. Recommandations2007 : [On-line] Disponible sur : http://www.has-sante.fr.

20. Deccache A. Aider le patient à apprendre sa santé et sa maladie : ce que nous apprennent l'évolution de l'éducation thérapeutique et ses développements psychosociaux. Med Hyg 2004;2484:1168-72.

21. Taylor V. Health education-a theorical mapping. Health Educ J 1990;49(1).

22. Szasz T, Hollender M. The basic models of the doctorpatient relations. 1976;97:585-9.

23. Deccache A, Lavenhomme E. Information et éducation du patient. Bruxelles : De Boeck université ; 1989.

24. Bélair L. La formation à la complexité du métier d'enseignement. In : Paquay L, Altet M, Charlier E, Perrenoud P, editors. Former des enseignants professionnels Quelles stratégies ? Quelles compétences? Bruxelles : De Boeck, Perspectives en éducation ; 1996:63-75.

25. Jouquan J, Bail P. À quoi s'engage-t-on en basculant du paradigme d'enseignement vers le paradigme d'apprentissage ? Pédagogie Médicale 2003;4:16375.

26. De Ketele J-M, Roegiers X. Méthodologie du recueil d'informations : fondements de méthode d'observation, d'interviews et d'étude de documents. Bruxelles : De Boeck université ; 1991.

Correspondance et offprints: UCL-IRSS-RESO. Université Catholique de Louvain. Clos chapelle-aux-champs, 30 bte B.1.30.14, 1200 Bruxelles, Belgique.

Mailto : Sandrine.roussel@uclouvain.be 